\title{
Successful Embolization of a Large Vein of Galen Malformation in a Premature Infant Presenting with Congestive Heart Failure and Persistent Pulmonary Hypertension
}

\author{
Ashraf M. Aly, M.D., Ph.D. ${ }^{1}$ Clarisa Y. Garcia, M.D. ${ }^{2}$ Rudiger von Ritschl, M.D. ${ }^{3}$ \\ ${ }^{1}$ Division of Pediatric Cardiology, Children's Hospital, Galveston, Texas \\ 2 Primary Children's Medical Center, Salt Lake City, Utah \\ 3 Division of Neuroradiology, Children's Hospital, 301 University \\ Boulevard, Galveston, Texas \\ Address for correspondence and reprint requests Ashraf M. Aly, \\ M.D., Ph.D., Director, Division of Pediatric Cardiology; Associate \\ Professor of Pediatrics, Department of Pediatrics, Children's Hospital, \\ 301 University Boulevard, Galveston, TX 77555-0367 \\ (e-mail: amaly@utmb.edu).
}

Am J Perinatol Rep 2012;2:19-22.

\author{
Abstract \\ Keywords \\ - vein of Galen \\ malformation \\ - high-output heart \\ failure \\ - endovascular \\ embolization
}

We report a case of a premature infant with a vein of Galen malformation (VGM) who presented with high-output heart failure, pulmonary hypertension, and respiratory distress. An echocardiogram showed normal cardiac anatomy, patent ductus arteriosus, and retrograde flow in the descending aorta. Ultrasonography and computed tomography of head confirmed the diagnosis of a large VGM. The patient developed multiorgan failure. Endovascular embolization of the VGM was successfully performed with an excellent outcome.
Vein of Galen malformation (VGM) was first described in 1895 as an abnormal fistula between the cerebral arteries and the great vein of Galen. ${ }^{1,2}$ Neonates with VGM usually present with cardiac failure and cyanosis, and can be mistaken as having cyanotic congenital heart diseases. Without intervention the mortality rate among infants with VGM is close to $100 \%{ }^{3}$ Endovascular embolization revolutionized the management of VGM and significantly reduced its morbidity and mortality.

\section{Case Report}

The patient was a 3280-gram newborn female born at 35 weeks gestation. Apgar scores were 7 and 9 at 1 and 5 minutes, respectively. The patient developed early respiratory distress, mild cyanosis $\left(\mathrm{O}_{2}\right.$ saturation $88 \%$ ), and required endotrachial intubation. Initial cardiac examination showed hyperdynamic precordium and a faint systolic murmur. Chest X-ray showed cardiomegaly and diffuse opacification of both lungs. Surfactant and nitric oxide were administered with the assumption that the patient had respiratory distress syndrome and persistent pulmonary hypertension of the newborn.

The patient developed increased tachycardia (heart rate of 195/minutes), metabolic acidosis, and decreased urine output. An audible bruit was heard upon head auscultation. An echocardiogram showed normal cardiac anatomy, hyperdynamic function (systolic function [SF] 55\%), and a large patent ductus arteriosus (PDA) with a bidirectional shunt flow. There was retrograde flow in the distal aortic arch to the dilated neck vessels (-Fig. 1) and pulmonary hypertension (65 $\mathrm{mm} \mathrm{Hg}$ ). Ultrasonography and computed tomography of head confirmed the presence of a large VGM $(2.81 \times 1.65 \mathrm{~cm})$ with high velocity turbulent flow.

The renal and cardiac function deteriorated (SF 25\%), and a strain pattern was noted in electrocardiogram (EKG). On the third day of life, angiography revealed a large VGM (-Fig. 2A). Transarterial embolization was performed using Guglielmi detachable coils, resulting in a substantial decrease in VGM flow. received

June 7, 2011

accepted after revision

October 6, 2011

published online

January 30, 2012
Copyright $\odot 2012$ by Thieme Medical Publishers, Inc., 333 Seventh Avenue, New York, NY 10001, USA. Tel: +1(212) 584-4662.
DOI http://dx.doi.org/ 10.1055/s-0031-1300966. ISSN 2157-6998. 


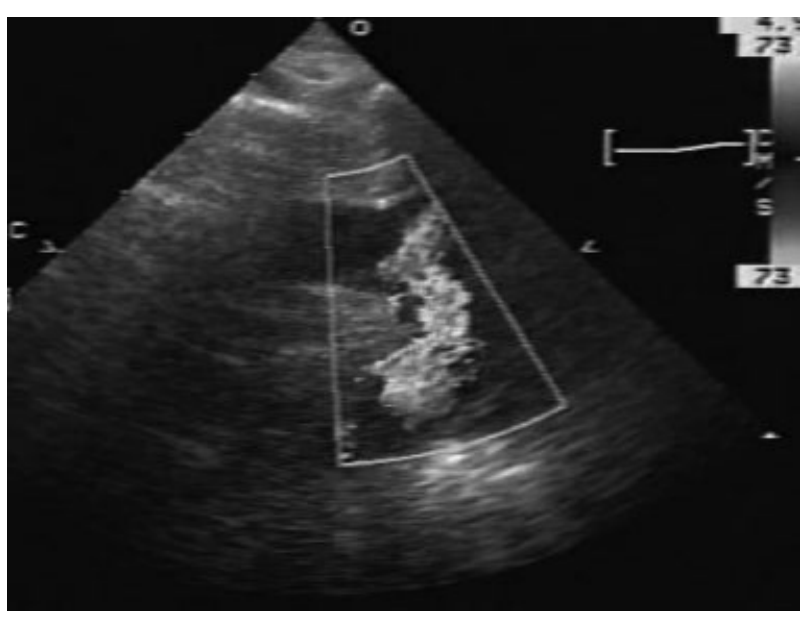

Figure 1 Doppler of the aortic arch showing retrograde flow in the descending aorta.

Initial clinical improvement was achieved, but the renal function continued to decline. On the ninth day of life, the VGM was reassessed and transvenous embolization was performed. Postembolization angiogram showed a significant decrease in shunting through the VGM and improved cortical artery flow (-Fig. 2B).

The hospital course which lasted 2.5 months was complicated by preprocedural clinical sepsis, renal failure, and persistent PDA requiring surgical ligation, and postprocedural seizures, chylothorax, pericardial effusion, and osteomyelitis of the right femur. Serial electroencephalograms (EEGs) showed improvement and the last EEG before discharge had no seizure activity. The chylothorax was treated with chest tube drainage, Portagen formula (Mead Johnson Nutrition, Glenview, IL), and octreotide. ${ }^{4}$

The patient did remarkably well despite of all these complications. At 26 months, she was seizure free, had a normal physical examination and age appropriate milestones. An echocardiogram showed normal cardiac anatomy and function.

\section{Discussion}

VGM is a rare anomaly of the cerebral vasculature that results in significant aneurysmal dilation of the great vein of Galen. The clinical presentation of VGM varies with age. Neonates usually have multiple fistulae and present in high-output cardiac failure. ${ }^{5}$ Infants and younger children have a single fistula and present with seizures, hydrocephalus, distention of scalp veins, and failure to thrive. Older children and adults often present with neurological symptoms of headache and seizure. ${ }^{6}$ Due to collateral circulation, there is an increased blood flow in the pial veins leading to subarachnoid and intracranial hemorrhage. $^{7}$

High-output cardiac failure in neonates with VGM is multifactorial in origin and responds poorly to medical management. During fetal life, the low resistance uteroplacental circulation diminishes the flow through the cerebral circulation. After birth, the placental circulation is lost and a significant portion of the cardiac output is directed toward the VGM. There is a compensatory increase in cardiac output and blood volume to maintain adequate systemic perfusion. This causes significant tachycardia, hyperdynamic cardiac function, and a wide pulse pressure. Since cardiac perfusion is dependent on the diastolic time and pressure, the cardiac function eventually deteriorates and ischemic changes can be noted in EKGs. The perfusion of all organs "including the brain," is further compromised by stealing of blood from the systemic circulation by the low resistance VGM.

The management of VGM progressed through three historical eras. The first era involved the earliest descriptions

A
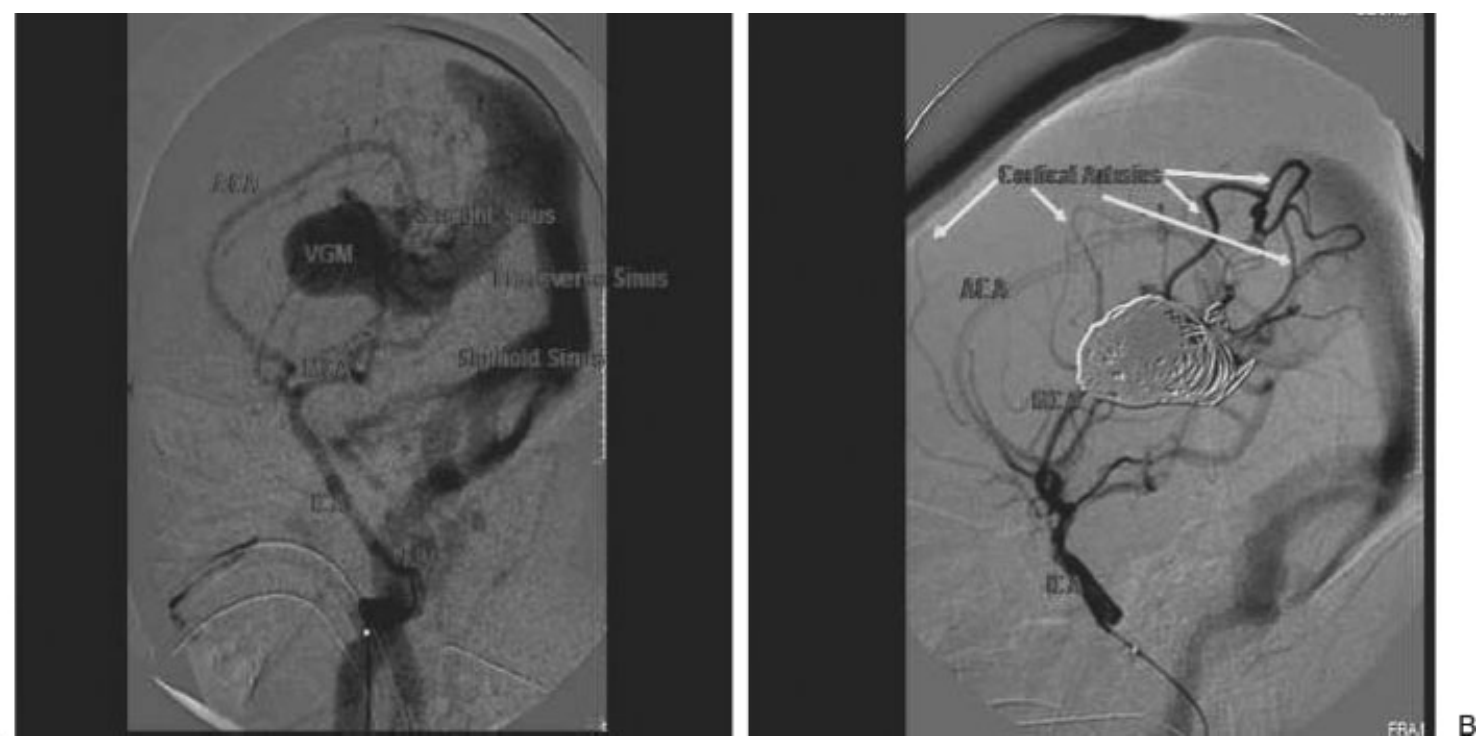

Figure 2 Angiograms of the vein of Galen malformation and the feeder arteries (A) pre-embolization and (B) postembolization, with improvement in filling of cortical arteries. 
of pathology, anatomy, syndromes, and case reports. No interventions were offered to neonates with VGM and the mortality was close to $100 \%{ }^{3}$

During the second era, previously described syndromes were correlated with their outcomes. The main intervention was a combined open microsurgery through craniotomy and a direct transtrocular embolization, but mortality and morbidity remained as high as $91 \%$ regardless of surgery or medical management. ${ }^{8}$

The third and current phase is the neurointervention era. The development of intravascular occlusion devices has shown to be safer and more effective. A comparison study showed death of all six neonates with VGM who underwent direct microsurgery while six of the eight who were treated with endovascular embolization survived. ${ }^{9}$ In a case series of 36 VGMs treated with endovascular embolization, the mortality rate was $13 \%{ }^{10}$

Neonatal VGM remains a serious condition that requires early diagnosis and a multidisciplinary approach of management and intervention. Coordination among neonatologist, pediatric cardiologist, and interventional neuoradiologist is essential for better patient care and outcome. Prematurity and the presence of multiorgan failure should not be considered absolute contraindications for early intervention if the patient can be stabilized for the procedure.

\section{References}

1 Blount JP, Oakes WJ, Tubbs RS, Humphreys RP. History of surgery for cerebrovascular disease in children. Part II. Vein of Galen malformations. Neurosurg Focus 2006;20(6):E10

2 Hoffman HJ, Chuang S, Hendrick EB, Humphreys RP. Aneurysms of the vein of Galen. Experience at The Hospital for Sick Children, Toronto. J Neurosurg 1982;57(3):316-322

3 Wong FY, Mitchell PJ, Tress BM, Dargaville PA, Loughnan PM. Hemodynamic disturbances associated with endovascular embolization in newborn infants with vein of Galen malformation. J Perinatol 2006;26(5):273-278

4 Helin RDDO, Angeles ST, Bhat R. Octreotide therapy for chylothorax in infants and children: a brief review. Pediatr Crit Care Med 2006;7(6):576-579

5 Gupta AK, Varma DR. Vein of Galen malformations: review. Neurol India 2004;52(1):43-53

6 Gold A, Ransohoff J, Carter S. Vein of Galen malformation. Acta Neurol Scand Suppl 1964;40(Suppl 11):1-31

7 Berenstein A, Lasjaunias P. Arteriovenous fistulas of the brain. In: Surgical Neuroangiography 4. Endovascular Treatment of Cerebral Lesions. New York: Springer-Verlag; 1992:268-319

8 Johnston IH, Whittle IR, Besser M, Morgan MK. Vein of Galen malformation: diagnosis and management. Neurosurgery 1987; 20(5):747-758

9 Ciricillo SF, Edwards MS, Schmidt KG, et al. Interventional neuroradiological management of vein of Galen malformations in the neonate. Neurosurgery 1990;27(1):22-27, discussion 27-28

10 Lasjaunias P, Rodesch G, Terbrugge K, et al. Vein of Galen aneurysmal malformations. Report of 36 cases managed between 1982 and 1988. Acta Neurochir (Wien) 1989;99(1-2):26-37 